\title{
Pathologic complete response and outcomes by intrinsic subtypes in NSABP B-41, a randomized neoadjuvant trial of chemotherapy with trastuzumab, lapatinib, or the combination
}

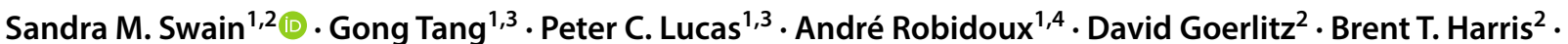 \\ Hanna Bandos $^{1,3}$. Charles E. Geyer Jr. ${ }^{1,5}$. Priya Rastogi ${ }^{1,3,6}$. Eleftherios P. Mamounas ${ }^{1,7} \cdot$ Norman Wolmark $^{1,3}$
}

Received: 5 July 2019 / Accepted: 4 August 2019 / Published online: 19 August 2019

(C) The Author(s) 2019

\begin{abstract}
Purpose NSABP B-41, a phase three randomized trial, evaluated neoadjuvant lapatinib, trastuzumab, or the combination with chemotherapy in patients with HER2-positive operable breast cancer. Though no significant difference in pathologic complete response (pCR) was found among the three arms, pCR was associated with prolonged survival. We analyzed tumor intrinsic subtypes with Prediction Analysis of Microarray 50 in a subset of B-41 patients to determine their value in predicting HER2-targeting benefit.

Methods Pearson's Chi square test and logistic regression were used to compare pCR in the breast and nodes (ypT0/Tis ypN0). Kaplan-Meier estimates and Cox models were used to compare event-free and overall survival among subtypes.

Results Intrinsic subtypes were determined in 271 baseline core biopsy samples. The pCR rate among patients with HER2enriched (HER2E) subtype was greater compared to other subtypes combined (120/197, 60.9\% versus 19/74, 25.7\%; $p<0.001$ ). In multivariate analysis among patients receiving trastuzumab-containing regimens (with clinical factors and HER2E subtype as factors), HER2E subtype was most strongly associated with pCR [OR 8.41 (95\% CI 2.52-28.1) $p<0.001$ ]. Patients with HER2E tumors did not benefit more from dual HER2-targeted therapy versus trastuzumab. The pCR rate was higher among HER2E tumors versus other subtypes in both estrogen receptor-positive and -negative tumors $(p \leq 0.001)$. Higher ESRl gene expression was associated with lower pCR rate. No association was observed between subtype and longterm outcomes.

Conclusion Patients with HER2E tumors were most likely to attain pCR versus other subtypes. HER2E subtype represents a favorable marker for predicting HER2-targeting benefit, particularly with trastuzumab-based therapies.
\end{abstract}

Keywords Breast cancer $\cdot$ HER2 enriched $\cdot$ Intrinsic subtype $\cdot$ Genomic $\cdot$ Neoadjuvant $\cdot$ Trastuzumab

This work was previously presented as a poster at the 54th Annual Meeting of the American Society of Clinical Oncology, Chicago, IL, June 1-5, 2018. Abstr. LBA501.

Electronic supplementary material The online version of this article (https://doi.org/10.1007/s10549-019-05398-3) contains supplementary material, which is available to authorized users.

Sandra M. Swain

sandra.swain@georgetown.edu

Extended author information available on the last page of the article

\section{Introduction}

Survival is increased in the adjuvant and metastatic settings with human epidermal growth factor 2 (HER2)-targeted treatment in patients with HER2-positive breast cancer [1,2]. A meta-analysis of neoadjuvant trials demonstrated that pathologic complete response (pCR) in HER2-positive tumors is most closely associated with favorable survival [3].

Randomized phase three trials have demonstrated heterogeneity in the $\mathrm{pCR}$ benefit of combining neoadjuvant lapatinib plus trastuzumab compared to trastuzumab alone when added to chemotherapy [4-6]. However, two meta-analyses of phase two and three trials demonstrated a statistically significant pCR benefit for the combination compared to trastuzumab alone [7, 8]. The National Surgical Adjuvant Breast and Bowel Project 
(NSABP) protocol B-41 was a three-arm randomized phase three trial that evaluated neoadjuvant trastuzumab, lapatinib, or both in patients receiving chemotherapy [5]. The pCR rate in breast and nodes (total pCR; ypN0/Tis ypNO) for each arm was $49.4 \%, 47.4 \%$, and $60.2 \%$, respectively (combination versus trastuzumab, $p=0.056$ ). Although the combination was not superior, subgroup analysis suggested that patients with positive clinical nodal status at baseline achieved a significantly higher pCR rate with the combination compared to trastuzumab alone. The 5-year recurrence-free interval (RFI; time from surgery to local, regional, or distal recurrence) and overall survival (OS) did not show a significant benefit for either lapatinib or the combination versus trastuzumab [9]. However, exploratory analysis suggested that trastuzumabcontaining regimens are superior to lapatinib alone in longterm outcomes (overall log-rank $p=0.05$, RFI; $p=0.07$, OS). Importantly, breast $\mathrm{pCR}$ was significantly associated with improved RFI $(p=0.0009)$ and OS $(p=0.0004)$.

Tumor intrinsic subtype, as measured by a 50-gene intrinsic subtype profile Prediction Analysis of Microarray 50 (PAM50), provides prognostic and predictive information beyond conventional determinants of hormone receptor (HR) and HER2 status in breast cancer [10]. The HER2-enriched (HER2E) breast cancer intrinsic subtype is predictive of attaining $\mathrm{pCR}$ in patients treated with lapatinib, trastuzumab, or the combination added to neoadjuvant chemotherapy [6, 11-13]. More precisely defining the use of HER2E to determine patients who benefit from single or dual HER2-targeted neoadjuvant therapy with or without chemotherapy is essential for clinical application.

The goal of this study is to determine whether intrinsic subtype by PAM50 can predict pCR and long-term outcomes in NSABP B-41. We hypothesized that the benefit of dual versus single HER2 targeting is limited to the HER2E subtype. This report adheres to REMARK criteria (Suppplementary material Table S6) [14].

\section{Materials and methods}

\section{Study design and patients}

NSABP B-41 was an open-label, three-arm phase three study performed from 2007 to 2011. Eligibility criteria and trial procedures have been previously reported [5]. Tumors were locally tested for HER2. For the current preplanned secondary analyses of B-41, any patient with an available baseline core biopsy sample, who had pCR ascertained, and who did not withdraw consent was eligible.

The primary objective of the current analyses was to determine the value of genomic subtypes as measured by PAM50 in predicting the chance of pCR among patients with HER2-positive tumors receiving neoadjuvant
HER2-targeting regimens. Pathologic complete response was defined as the absence of any invasive component in the resected breast specimen and absence of cancer on hematoxylin and eosin (H\&E) evaluation of all resected lymph nodes following completion of neoadjuvant therapy (ypT0/ Tis ypN0) [3]. Secondary objectives included comparing patients with HER2E tumors to those with other subtypes in long-term outcomes (event-free survival [EFS] and OS) and determining whether patients with HER2E tumors benefitted more from dual versus single HER2-targeting regimens versus those with other subtypes in pCR, EFS, and OS. Event-free survival was defined as time from randomization to first local, regional, or distant recurrence, second primary, or death from any cause. Overall survival was defined as the time from randomization to death from any cause. Since lapatinib-alone regimens were shown to be numerically inferior to trastuzumab-containing regimens in the literature [4, 5], trastuzumab-containing arms were analyzed in these secondary analyses.

The clinical data are housed at the NSABP Biostatistical Center in Pittsburgh Pennsylvania. Since the data were anonymized to Georgetown investigators, a waiver from the Georgetown institutional review board was obtained to proceed with the study.

\section{Procedures}

For each patient, serial $10 \mu \mathrm{m}$ sections were cut from selected tissue blocks by the NSABP Department of Pathology. Unstained sections of formalin-fixed paraffin-embedded (FFPE) tumor samples and associated H\&E stained slides were sent to the Genomic and Epigenomics Shared Resource (GESR) at Georgetown University Medical Center (GUMC) blinded to all clinical data. These samples underwent pathological examination to confirm diagnosis and identify malignant tissue in the GUMC Histopathology and Tissue Shared Resource (HTSR) to guide subsequent RNA isolation. Areas with tumor were microdissected from those slides using the H\&E slides as templates. Total RNA was extracted from the microdissected tissues after deparaffinization using the Roche High Pure FFPET RNA Isolation Kit (Roche Molecular Systems, Pleasanton, CA) per manufacturer's instructions. The RNA quantity was estimated with ultraviolet-visible spectrophotometry using the NanoDrop 1000 spectrophotometer (Thermo Fisher Scientific, Waltham, MA) to ensure sample purity (optical density 260/280 nm ratio 1.7-2.5). To assess RNA quality, samples were analyzed using the Agilent RNA 6000 Nano Kit and Agilent 2100 Bioanalyzer (Agilent, Santa Clara, CA). Degree of RNA integrity was assessed using the smear analysis function in the Agilent 2100 Expert Software to measure the percentage of RNA molecules $>300 \mathrm{bp}$. Final 
RNA concentration $(12.5 \mathrm{ng} / \mu \mathrm{L})$ was normalized across all samples before input.

From each sample, $150 \mathrm{ng}$ of RNA was hybridized to the 72-plex human PAM50-research use only (PAM50RUO) CodeSet (Prosigna ${ }^{\mathrm{TM}}$ ) and processed on the nCounter Sprint Profiler (NanoString Technologies, Seattle, WA) according to manufacturer protocols. The system uses sequence-specific probes that hybridize directly to the mRNA in solution: a reporter probe, which carries a targetspecific, four-color, six-position fluorescent barcode, and a capture probe, which allows the complex to be immobilized for data collection. The PAM50 assay (using the PAM50RUO CodeSet) simultaneously measures the expression levels of 50 target sequences, including eight endogenous invariant mRNA targets, six positive quality control targets, and eight negative quality control targets consisting of probes with no sequence homology to human RNA (Supplementary material Table S7) [15].

\section{Statistical methods}

The Pearson Chi square test was used to compare treatment and stratification factors between patients who had PAM50 subtype determined from a core biopsy sample to the remaining B- 41 patients. For the primary analysis, the Pearson's Chi square test with continuity correction was used to test whether patients with HER2E tumors achieved a higher pCR rate than patients with other subtyped tumors. The Cochran-Mantel-Haenszel test was also performed with treatment as the stratification factor. Logistic regression models were used to test whether tumor subtype (HER2E versus others) is predictive of pCR in patients on trastuzumab-containing regimens with adjustment for clinical factors of age ( $\geq 50$ versus $<50$ years), HR status (positive versus negative), clinical nodal status (positive versus negative), clinical tumor size ( $\geq 4.1 \mathrm{~cm}$ versus $2-4 \mathrm{~cm}$ ), and treatment (trastuzumab plus lapatinib versus trastuzumab alone). Interaction between treatment and subtype was tested in the multivariate logistic regression model. The Breslow-Day test was also used to analyze whether patients with HER2E tumors benefitted more from dual-targeted therapy versus trastuzumab alone in $\mathrm{pCR}$.

In the secondary analyses of EFS and OS, the log-rank test was used to test whether patients with HER2E tumors achieved better EFS or OS than patients with other subtyped tumors. Cox proportional hazards models were used to test whether tumor subtype (HER2E versus others) was independently predictive of EFS or OS with adjustment for treatment and HR status (positive/negative). Separate Cox models, based on data from patients receiving trastuzumab-based regimens, were used to test whether an interaction exists between tumor subtype (HER2E versus others) and dual targeting (trastuzumab plus lapatinib versus trastuzumab), and an interaction between the expression level of ERBB2, $E S R 1$, and dual targeting. To study the prognostic utility of individual genes on $\mathrm{pCR}$ in patients receiving trastuzumabcontaining regimens, univariate logistic regression models were used with the Benjamini-Hochberg procedure to control the false discovery rate (FDR) at 0.1 [16]. The Holm's step-down procedure was used to identify gene signatures significantly prognostic for $\mathrm{pCR}$ with a familywise error rate (FWER) controlled at 0.05 [17]. Exploratory multivariate logistic regression models were used to predict $\mathrm{pCR}$ with clinical factors, the selected individual genes, and tumor subtype combined. Model selection was done via likelihood ratio test between the nested model and a sub-model, and the Akaike information criterion between non-nested models [18].

\section{Results}

A total of 329 tissue samples from 276 patients enrolled in B-41 were available, including 276 baseline samples from core biopsy prior to neoadjuvant regimens and 53 tissue samples (47 breast tissue and 6 lymph node) obtained at surgery after neoadjuvant treatment (Fig. 1). Twelve samples, four from core biopsy and eight breast tissue samples from surgery, either did not have tumor or had $<10 \%$ tumor cells in the available sections and were not assayed. Among the remaining baseline 272 core biopsy samples, intrinsic subtype was successfully determined in 271 . Subsequent analyses are based on data from these patients. Comparison of baseline characteristics between the patients included and not included in the analysis showed no differences except for a higher proportion of estrogen receptor (ER)-negative cases in the included cohort $(p=0.01$; Table 1$)$.

\section{PCR by intrinsic subtype}

In the core biopsy intrinsic subtype analysis, pCR was achieved in 120 of 197 patients (60.9\%, 95\% CI 53.7-67.3\%) with HER2E, 10 of $26(38.5 \%, 95 \%$ CI 20.4-56.3\%) with basal-like, 3 of $23(13.0 \%, 95 \%$ CI 3.3-29.7\%) with luminal A, and 6 of $25(24.0 \%, 95 \%$ CI 9.8-41.7\%) with luminal B subtype (Fig. 2a). The pCR rate among patients with HER2E subtype was statistically significantly greater compared to other subtypes combined [60.9\% vs. $25.7 \%$ (95\% CI 16.4-36.0\%); $p<0.001$ ].

All three HER2-targeted neoadjuvant regimens produced higher pCR rates in the HER2E population as compared to other intrinsic subtypes (Table 2; Fig. 2B). The odds ratio (OR) for pCR associated with HER2E (versus other subtypes) among patients treated with trastuzumab was 8.7 (95\% CI 2.7-28.5). The corresponding OR for pCR among 


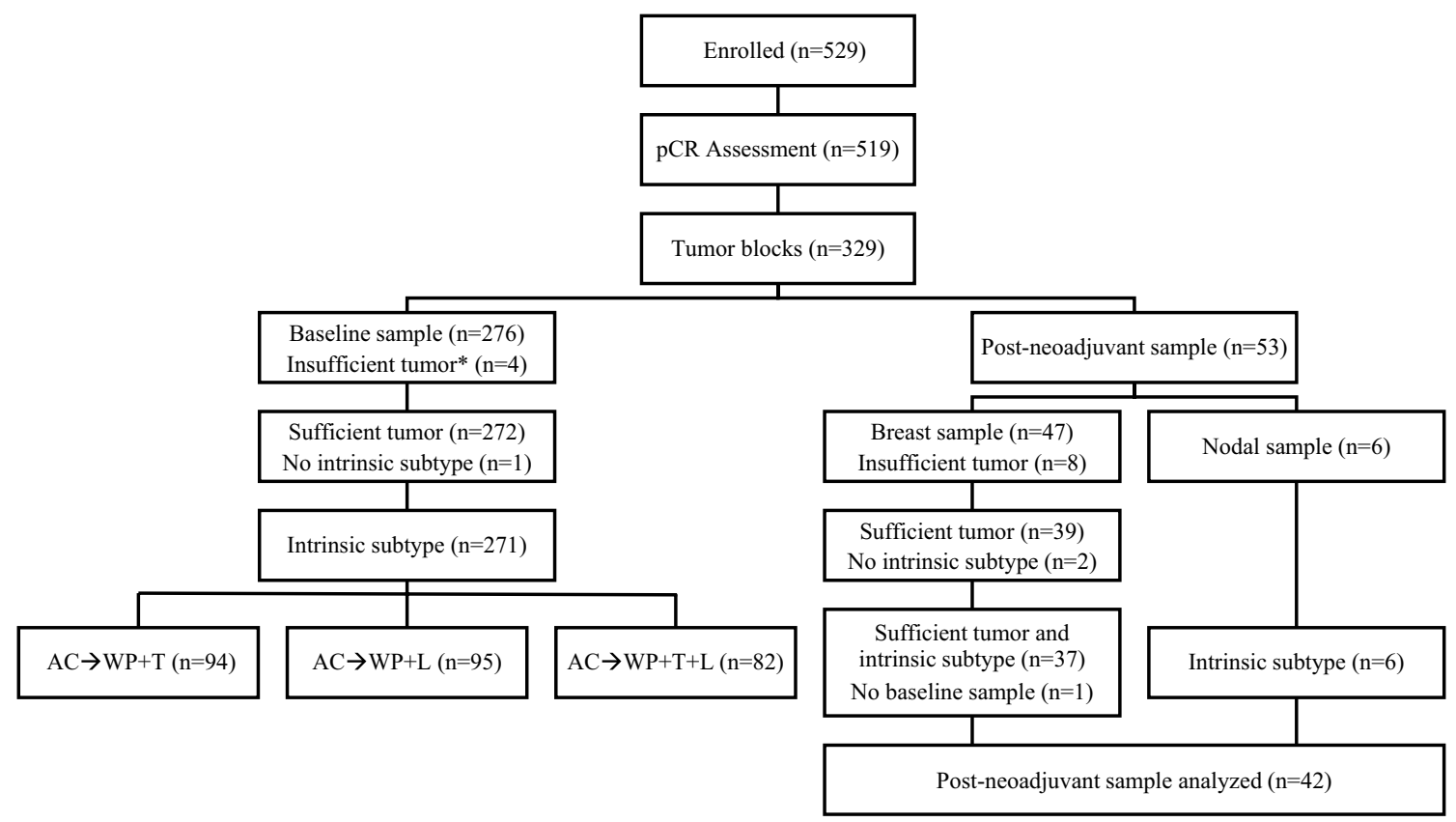

Fig. 1 NSABP B-41 patient sample flowchart. *Samples either did not have tumor or had $<10 \%$ tumor cells in the available sections and were not assayed. $A C$ doxorubicin and cyclophosphamide, $L$ lapatinib, $p C R$ pathologic complete response, $T$ trastuzumab, $W P$ weekly paclitaxel

Table 1 Baseline characteristics of patients included and excluded in the current analysis

\begin{tabular}{|c|c|c|c|c|}
\hline Category & Characteristic & $\begin{array}{l}\text { Included }(N=271), \\
N(\%)\end{array}$ & $\begin{array}{l}\text { Excluded } \\
(N=258), N(\%)\end{array}$ & $p$ value $^{\mathrm{a}}$ \\
\hline \multirow[t]{3}{*}{ Treatment } & $\mathrm{AC} \rightarrow \mathrm{WP}+\mathrm{T}$ & $94(35)$ & $87(34)$ & \multirow[t]{3}{*}{0.37} \\
\hline & $\mathrm{AC} \rightarrow \mathrm{WP}+\mathrm{L}$ & $95(35)$ & $79(31)$ & \\
\hline & $\mathrm{AC} \rightarrow \mathrm{WP}+\mathrm{TL}$ & $82(30)$ & $92(36)$ & \\
\hline \multirow[t]{2}{*}{ Clinical nodal status } & No & $130(48)$ & $127(49)$ & \multirow[t]{2}{*}{0.52} \\
\hline & $\mathrm{N} 1+$ & $141(52)$ & $131(51)$ & \\
\hline \multirow[t]{2}{*}{ Age } & $<50$ years & $135(50)$ & $142(55)$ & \multirow[t]{2}{*}{0.23} \\
\hline & $\geq 50$ years & $136(50)$ & $116(45)$ & \\
\hline \multirow[t]{2}{*}{ Clinical tumor size } & $2.0-4.0 \mathrm{~cm}$ & $136(50)$ & $135(52)$ & \multirow[t]{2}{*}{0.62} \\
\hline & $4.1+\mathrm{cm}$ & $135(50)$ & $123(48)$ & \\
\hline \multirow[t]{2}{*}{ Hormone receptor status } & Negative & $116(43)$ & $82(32)$ & \multirow[t]{2}{*}{0.01} \\
\hline & Positive & 155 (57) & $176(68)$ & \\
\hline
\end{tabular}

$A C$ doxorubicin and cyclophosphamide, $L$ lapatinib, $T$ trastuzumab, $W P$ weekly paclitaxel

${ }^{\text {a }}$ Pearson Chi square test patients treated with trastuzumab plus lapatinib was 7.5 (95\% CI 2.5-21.9).

Among patients treated with trastuzumab-containing neoadjuvant regimens, HER2E subtype was statistically significantly associated with higher pCR rate (CochranMantel-Haenszel test $p<0.0001)$. In multivariate logistic regression analysis for $\mathrm{pCR}$ among patients receiving trastuzumab-containing regimens $(n=176)$ that included clinical factors and HER2E subtype as factors, HER2E subtype was strongly associated with pCR (OR 8.41 [95\% CI 2.52-28.1] $p<0.001$; Table 3). The test for interaction between dual targeting and HER2E subtype was not statistically significant $(p=0.94)$. The $p$ value for the Breslow-Day test on homogeneity of the ORs is 0.85 .

The HR status of tumors according to intrinsic subtype is shown in Fig. 3. Of HER2E tumors, 105 (53.3\%) were HRpositive and 100 (50.8\%) were ER-positive. According to ER status, the proportions of patients achieving pCR were $69.1 \%$ (67/97, 95\% CI 58.8-77.2\%) for HER2E and ER-negative tumors, 53.0\% (53/100, 95\% CI 42.8-62.2\%) for HER2E and ER-positive tumors, $29.2 \%$ (7/24, 95\% CI 13.0-47.6\%) for ER- negative tumors of other subtypes combined, and $24.0 \%$ 


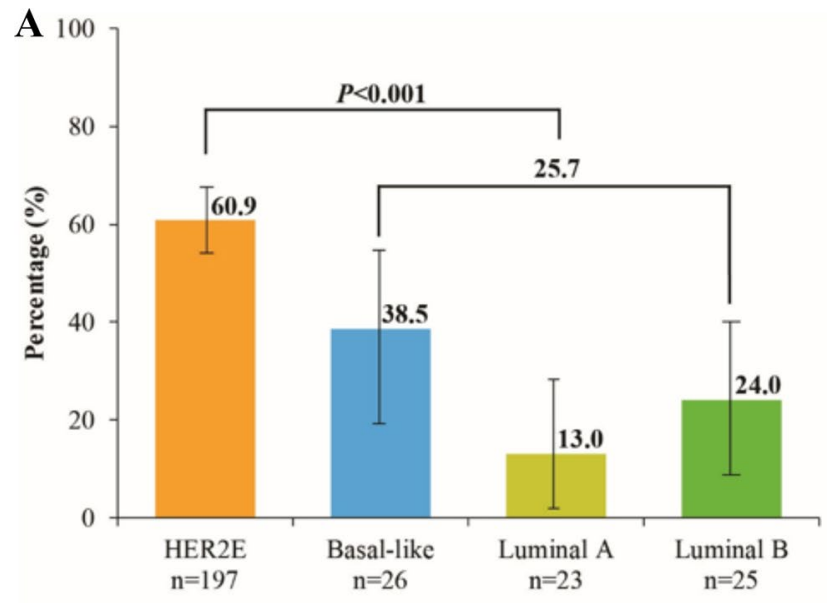

B

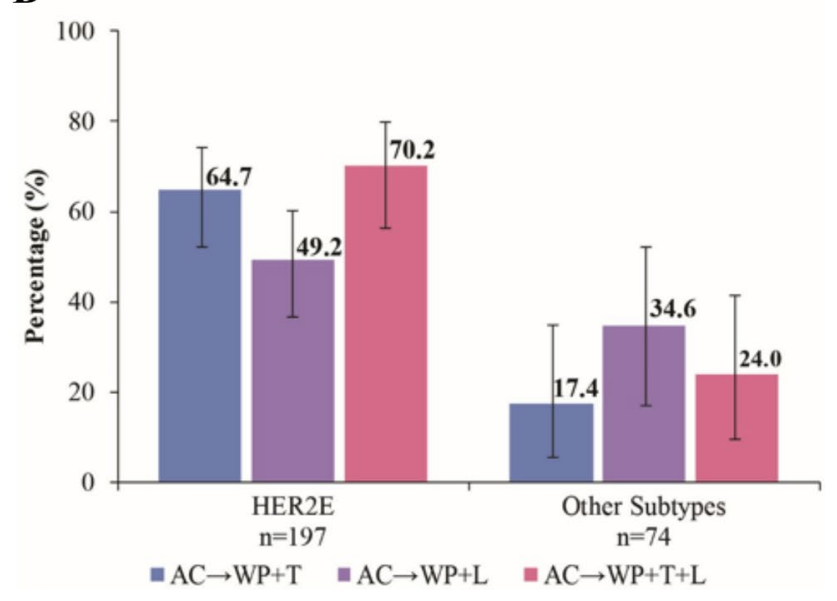

Fig. 2 Total pCR (ypT0/Tis ypN0) by core biopsy intrinsic subtype. (A) Total pCR between HER2E and other subtypes and (B) total pCR by treatment and intrinsic subtype. Vertical bars represent $95 \%$ confidence intervals. $A C$ doxorubicin and cyclophosphamide; HER $2 E$ HER2-enriched, $L$ lapatinib, $T$ trastuzumab, $W P$ weekly paclitaxel in both ER-positive $(p=0.001)$ and ER-negative $(p<0.001)$ subgroups.

\section{pCR by gene expression analysis}

In patients on trastuzumab-containing regimens, 14 among 50 genes on the PAM50 panel were associated with pCR with FDR controlled at 0.1 . These genes were GRB7, ERBB2, PGR, MYC, ESR1, MAPT, BCL2, TMEM45B, CDC6, SLC39A6, CEP55, KIF2C, RRM2, and PHGDH (Table 4). Among these 14 genes, GRB7 (OR 1.58, adjusted $p=0.003$ ), ERBB2 (OR 1.6, adjusted $p=0.007$ ), $P G R$ (OR 0.71, adjusted $p=0.025$ ), and MYC (OR 0.57, adjusted $p=0.026$ ) were predictive of $\mathrm{pCR}$ with FWER controlled at 0.05 . Correlations among expression levels of these four genes were present with the Pearson correlation coefficients varying from 0.3 to 0.87 . Multivariable logistic regression models were used to combine clinical factors, such as ER status and nodal status; expression levels of GRB7, ERBB2, PGR, MYC, and ESR1; and the intrinsic subtype (HER2E versus others). Based on the Akaike information criterion, ESR 1 provided more prognostic power than ER status, and the intrinsic subtype was superior in prognostic value to $E R B B 2$ or $G R B 7$. In the final multivariable logistic regression model, statistically significant predictors of $\mathrm{pCR}$ were clinical node positivity (OR $0.50,95 \%$ CI $0.25-0.98, p=0.04$ ), ESR 1 expression (OR 0.86, 95\% CI 0.74-0.99, $p=0.04$, for doubling expression level), and HER2E subtype (OR 6.82, 95\% CI $2.98-15.5, p<0.0001)$. To assess the prognostic utility of nodal status, ESRI expression, and molecular subtype (HER2E versus others), patients on trastuzumab-based regimens were randomly split into a training set of 118 patients and a testing set of 58 patients. A multivariate logistic regression model was then fit to predict $\mathrm{pCR}$ with
Table 2 Pathologic complete response by treatment arm and intrinsic subtype (HER2E vs. others)

\begin{tabular}{lllcll}
\hline Treatment arm & Intrinsic subtype & $\mathrm{N}$ & $\mathrm{pCR}, \mathrm{N}(\%)$ & $95 \% \mathrm{CI}(\%)$ & $p$ value \\
\hline $\mathrm{AC} \rightarrow \mathrm{WP}+\mathrm{T}$ & HER2E & 71 & $46(64.7)$ & $52.5-74.6$ & $<0.001$ \\
& Others $^{\mathrm{a}}$ & 23 & $4(17.4)$ & $5.4-35.0$ & \\
$\mathrm{AC} \rightarrow \mathrm{WP}+\mathrm{L}$ & HER2E & 69 & $34(49.2)$ & $37.1-60.4$ & 0.29 \\
& Others $^{\mathrm{a}}$ & 26 & $9(34.6)$ & $17.5-52.5$ & \\
$\mathrm{AC} \rightarrow \mathrm{WP}+\mathrm{T}+\mathrm{L}$ & HER2E & 57 & $40(70.2)$ & $56.5-80.3$ & $<0.001$ \\
& Others $^{\mathrm{a}}$ & 25 & $6(24.0)$ & $9.8-41.7$ & \\
\hline
\end{tabular}

$A C$ doxorubicin and cyclophosphamide, HER2E human epidermal growth factor receptor 2-enriched, $L$ lapatinib, $p C R$ pathologic complete response, $T$ trastuzumab

${ }^{\mathrm{a}}$ Includes luminal A, luminal B, and basal-like
(12/50, 95\% CI 13.3-36.4\%) for ER-positive tumors of other subtypes combined. The pCR rate among HER2E tumors versus other subtypes was statistically significantly greater these three factors using data from the training set, and the resulting linear combination from the fitted logistic model was applied to data from patients in the testing 
Table 3 Multivariate logistic regression analysis of total $\mathrm{pCR}$ among patients treated with trastuzumab or trastuzumab plus lapatinib $(N=176)$

\begin{tabular}{llc}
\hline Factors & Odds ratio (95\% CI) & $p$ value $^{\mathrm{a}}$ \\
\hline Age $(\geq 50$ vs. $<50$ years) & $0.77(0.39-1.52)$ & 0.45 \\
Hormone receptor status (positive vs. negative) & $0.74(0.37-1.50)$ & 0.41 \\
Clinical nodal status (positive vs. negative) & $0.48(0.24-0.98)$ & 0.04 \\
Tumor size $(\geq 4.1$ vs. $2-4 \mathrm{~cm})$ & $1.28(0.64-2.55)$ & 0.48 \\
HER2E subtype & $8.41(2.52-28.1)$ & $<0.001$ \\
Dual targeting in HER2E tumors & $1.29(0.60-2.77)$ & 0.67 \\
Dual targeting in other subtypes & $1.37(0.33-5.74)$ & 0.52 \\
\hline
\end{tabular}

HER2E HER2-enriched, $p C R$ pathologic complete response

${ }^{\text {a}}$ Wald test

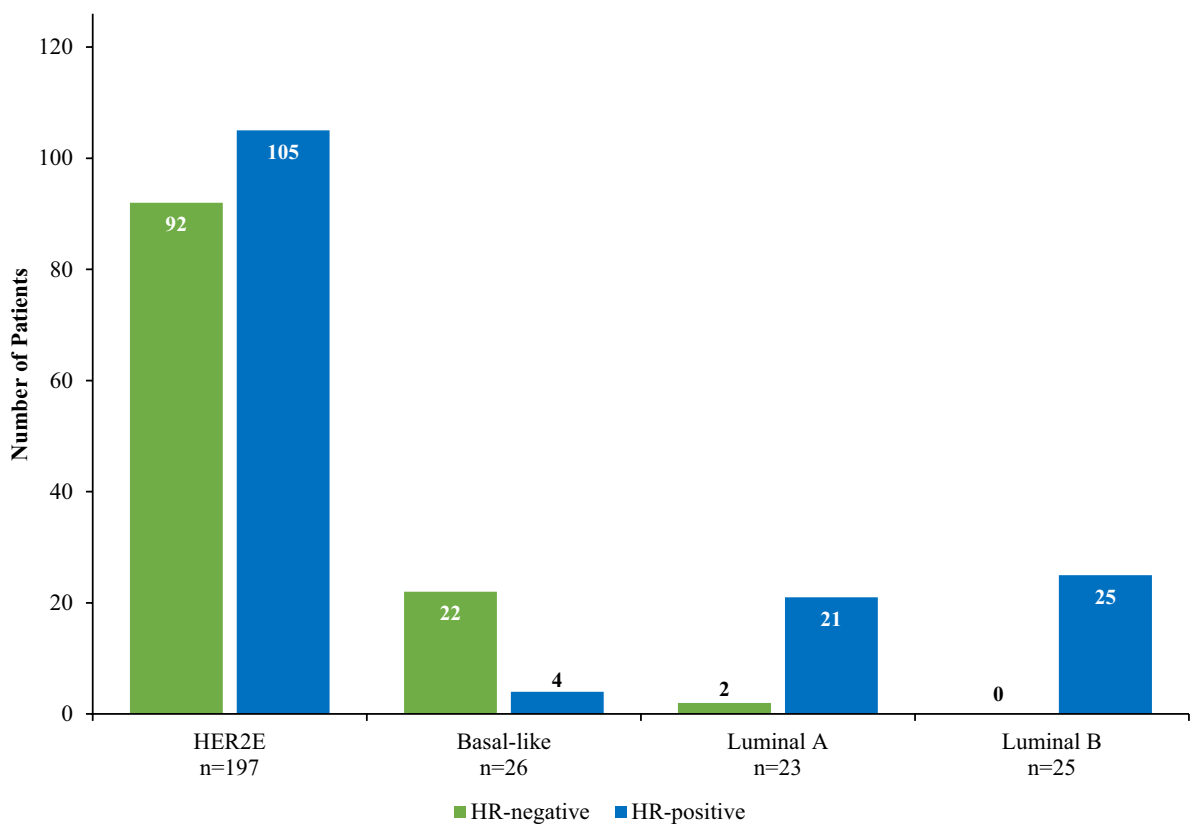

Fig. 3 HR status according to intrinsic subtype. HR-positive indicates estrogen receptor or progesterone receptor positive. HR-negative indicates estrogen receptor and progesterone receptor negative. HER $2 E$ HER2-enriched, $H R$ hormone receptor set as an index for $\mathrm{pCR}$. The resulting area under the receiver operating characteristic curve is 0.73 , indicating that molecular subtype and ESRl expression are strong and informative prognostic factors for $\mathrm{pCR}$ in patients receiving HER2-targeted therapy (Fig. 4).

\section{Residual disease}

Thirty-six patients with breast tissue samples and six patients with axillary node samples $(n=42)$ were analyzed for agreement in intrinsic subtype between core biopsy samples and residual breast tissues or lymph nodes at surgery (Table 5). Moderate agreement was observed [weighted Kappa statistic $=0.49(95 \%$ CI 0.30-0.67) .

\section{Long-term outcomes}

Among the 271 patients with core biopsy intrinsic subtype analysis, median follow-up was 5.1 years. A total of 48 EFS events occurred. Five-year EFS rates were 82.3\% (95\% CI $75.9-87.1 \%$ ) and $81.6 \%$ (95\% CI 70.2-88.9\%) for patients with HER2E and other subtypes, respectively. No statistically significant difference in EFS was observed between HER2E and other subtypes [hazard ratio $=0.69$ (95\% CI 0.26-1.81), log-rank $p=0.89$ ). The overall log-rank $p$ value for EFS among all subtypes was 0.28 (Fig. 5a). The Kaplan-Meier estimates of 5-year EFS rates by pCR and subtype are shown for patients who received trastuzumab-based regimens in Table 6. Among patients who did not achieve pCR, those with HER2E subtype had numerically worse prognosis than those with other subtypes. Conversely, among patients who achieved pCR, those with HER2E subtype had numerically better prognosis than those with other subtypes. 
Table 4 Individual gene signatures prognostic for total pCR among patients treated with trastuzumab or trastuzumab plus lapatinib $(n=176)$ with false discovery rate controlled at 0.1

\begin{tabular}{llll}
\hline Gene signatures & $\mathrm{OR}^{\mathrm{a}}$ & $p$ value $^{\mathrm{b}}$ & ${\text { Adjusted } p \text { value }^{\mathrm{c}}}^{\text {G }}$ \\
\hline ERB7 & 1.58 & $6.8 \mathrm{E}-5$ & 0.003 \\
PGBB2 & 1.60 & $1.5 \mathrm{E}-4$ & 0.007 \\
MYC & 0.71 & $5.2 \mathrm{E}-4$ & 0.025 \\
ESR1 & 0.57 & $5.5 \mathrm{E}-4$ & 0.026 \\
MAPT & 0.80 & 0.001 & 0.051 \\
BCL2 & 0.70 & 0.001 & 0.065 \\
TMEM45B & 0.67 & 0.004 & 0.19 \\
CDC6 & 1.41 & 0.006 & 0.25 \\
SLC39A6 & 1.40 & 0.008 & 0.32 \\
CEP55 & 0.71 & 0.008 & 0.34 \\
KIF2C & 1.91 & 0.01 & 0.40 \\
RRM2 & 1.60 & 0.02 & 0.63 \\
PHGDH & 1.64 & 0.02 & 0.68 \\
\hline
\end{tabular}

$p C R$ pathologic complete response

${ }^{\text {a } U n i v a r i a t e ~ l o g i s t i c ~ r e g r e s s i o n ~ m o d e l s ~}$

${ }^{\mathrm{b}}$ Univariate logistic regression models with total pCR as the outcome variable

${ }^{\mathrm{c}}$ Holm's step-down procedure to adjust for multiple testings

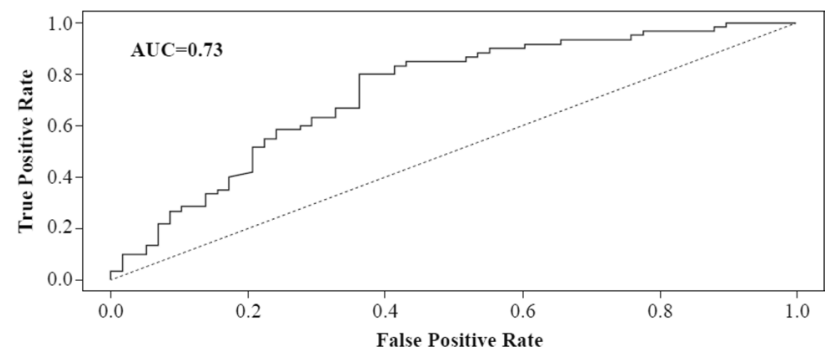

Fig. 4 Receiver operating characteristics curve for predicting total pCR among patients in the testing set $(n=58)$ based on the logistic regression model developed from the training set $(n=118)$. Involved predictors were clinical nodal status, ESRl gene expression, and subtype (HER2E vs. others). AUC area under the curve

Table 5 Correlation of intrinsic subtype between core biopsy and primary surgery ${ }^{\mathrm{a}}$

\begin{tabular}{llllll}
\hline \multirow{2}{*}{$\begin{array}{l}\text { Core biopsy } \\
\text { subtype }\end{array}$} & \multicolumn{5}{l}{ Surgical sample subtype, } \\
\cline { 2 - 6 } & HER2E & Basal-like & Luminal A & Luminal B & Total \\
\hline HER2E & 12 & 1 & 6 & 1 & 20 \\
Basal-like & 0 & 4 & 0 & 0 & 4 \\
Luminal A & 1 & 0 & 6 & 1 & 8 \\
Luminal B & 1 & 0 & 8 & 1 & 10 \\
Total & 14 & 5 & 20 & 3 & 42 \\
\hline
\end{tabular}

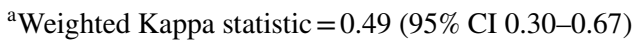

HER2E HER2-enriched
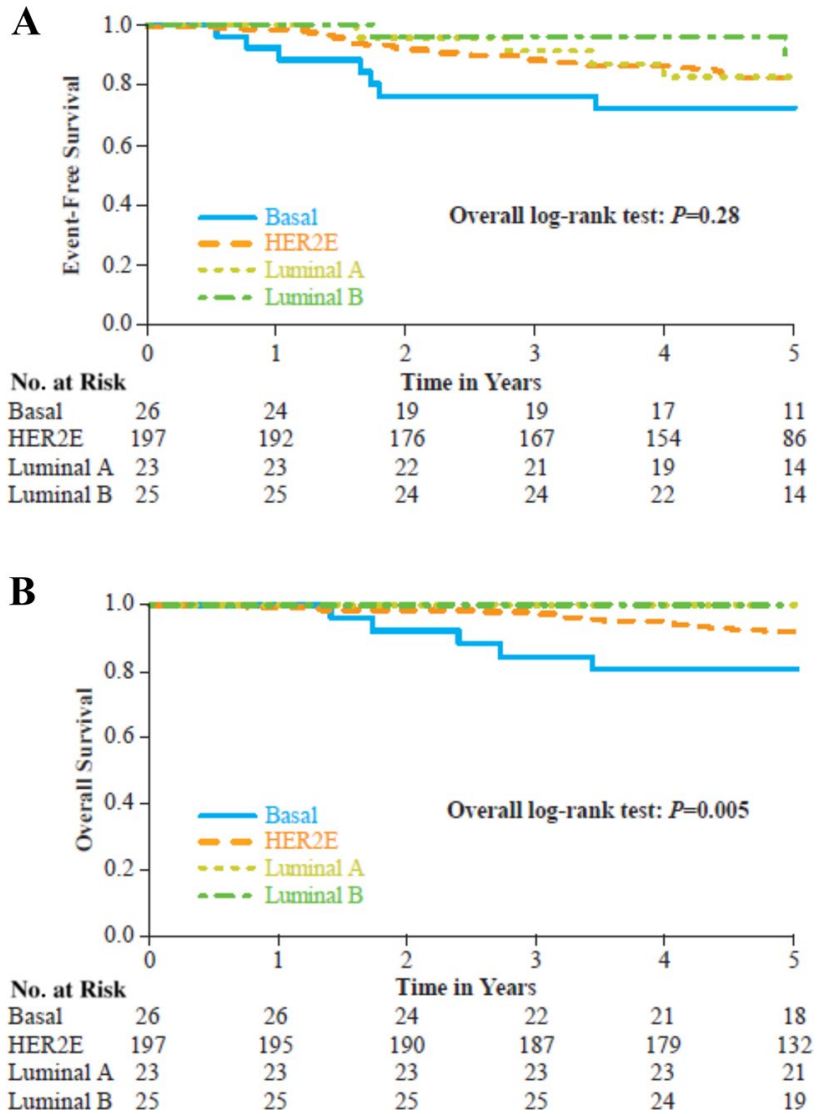

Fig. 5 Long-term outcomes. (A) Event-free survival and (B) overall survival. HER2E HER2-enriched

Table 6 Kaplan-Meier estimates of 5-year EFS by pCR and PAM50 subtype in patients treated with trastuzumab-containing neoadjuvant regimens

\begin{tabular}{llll}
\hline pCR status & PAM50 subtype & $\begin{array}{l}\text { No. of events/ } \\
\text { No. of } \\
\text { patients }\end{array}$ & $\begin{array}{l}\text { 5-year EFS (\%) (95\% } \\
\text { CI) }\end{array}$ \\
\hline No pCR & HERE & $12 / 42$ & $73.0(56.6,84.1)$ \\
& Other & $7 / 38$ & $81.5(65.1,90.7)$ \\
pCR & HER2E & $8 / 86$ & $90.1(81.2,94.9)$ \\
& Other & $2 / 10$ & $80.0(40.9,94.6)$ \\
\hline
\end{tabular}

EFS event-free survival, HER2E HER2-enriched, PAM50 prediction analysis of microarray $50, p C R$ pathologic complete response in breast and nodes (ypN0/Tis ypN0)

A total of 21 deaths occurred. Five-year OS rates were $92.1 \%$ (95\% CI $87.1-95.1 \%$ ) and $93.2 \%$ (95\% CI 84.5-97.1\%) for patients with HER2E and other subtypes, respectively. No statistically significant difference in OS was observed between HER2E and other subtypes [hazard ratio $=0.79(95 \%$ CI 0.41-1.52) $p=0.98]$. However, the overall log-rank $p$ value for OS among all subtypes was 0.005 (Fig. 5b), with shortened OS in the basal-like 
subgroup (5-year OS rate $80.8 \% ; p=0.01$ vs. HER2E subtype).

\section{Discussion}

This analysis of NSABP B-41 outcomes according to intrinsic subtypes as determined by PAM50 demonstrated that the HER2E subtype was statistically significantly associated with achieving $\mathrm{pCR}$ compared to other intrinsic subtypes in HER2-positive patients receiving trastuzumab-containing neoadjuvant regimens. This association was maintained regardless of ER status.

Selecting patients for HER2-targeted therapy remains a priority to ensure that those likely to benefit receive the most effective treatment and to avoid unnecessary costs and potential adverse events in patients unlikely to benefit. While current standard-of-care methods of determining HER2 positivity accurately identify a subset of patients who benefit from neoadjuvant HER2-targeted therapy, a substantial portion of HER2-positive patients fail to attain pCR $[4,19]$. Intrinsic subtype provides valuable information about the sensitivity of tumors to a variety of currently available agents including HER2-targeted agents, cytotoxic chemotherapy, and cyclin-dependent kinase inhibitors [10, 20]. Genomic analysis is now being evaluated for its ability to improve identification of patients who will respond to neoadjuvant HER2-targeted therapy [6, 11-13, 21]. Fumagalli, et al. demonstrated that among patients treated with neoadjuvant trastuzumab, lapatinib, or the combination with chemotherapy in NeoALTTO, the HER2E subtype was associated with a pCR rate of 52\% compared to $17-38 \%$ across other subtypes [13]. Similarly, Carey, et al. demonstrated a higher pCR rate among HER2E tumors compared to other subtypes in patients receiving neoadjuvant trastuzumab with or without lapatinib plus chemotherapy in CALGB 40601 [6]. Among all treatment arms, pCR rates were $66 \%, 34 \%$, and $34 \%$ for HER2E, luminal A, and luminal B subtypes, respectively. The current analysis of NSABP B-41 adds to this body of literature by demonstrating the benefit of intrinsic subtype as measured by PAM50 in predicting the benefit of HER2-targeting therapy. Patients with HER2E tumors were statistically significantly more likely to benefit from neoadjuvant HER2-targeted therapy added to chemotherapy than patients with basal-like, luminal A, or luminal B subtype tumors. The pCR rate in the HER2E subgroup was $61 \%$, consistent with NeoALTTO and CALGB40601. Conversely, pCR rates among patients in our analysis with other subtypes ranged from 13 to $38 \%$, consistent with pCR achieved with chemotherapy alone [22]. As such, whether HER2-targeted therapy offers benefit beyond chemotherapy or endocrine therapy in patients with HER2-positive disease and non-HER2E subtype requires investigation in future prospective clinical trials.

In a different approach, neoadjuvant trastuzumab plus lapatinib was compared to trastuzumab alone without chemotherapy in the single-arm PAMELA study [21]. Intrinsic subtype analysis also demonstrated an increased breast pCR rate among patients with HER2E tumors (41\%) compared to other subtypes (10\%). Although the breast pCR rate was lower than observed with regimens incorporating chemotherapy, these data provide a potential signal for eliminating chemotherapy in a subset of patients selected based on intrinsic subtype. A combined genomic marker incorporating HER2E and ERBB2-high mRNA was associated with increased pCR compared to other subtype and ERBB2low tumors when applied to data from 5 trials, including PAMELA, that evaluated single versus dual HER2-targeted therapy without chemotherapy in localized or advanced breast cancer. [23].

Prior data have demonstrated a lower pCR rate in response to neoadjuvant trastuzumab, lapatinib, or the combination among patients with HR-positive as compared to HR-negative disease [3, 4, 24]. In our analysis, pCR rates were $69.1 \%$ for HER2E/ER-negative tumors and $53.0 \%$ for HER2E/ER-positive tumors, suggesting that pCR rate remains decreased among HR-positive tumors within the HER2E subtype. However, the pCR rate among HER2E tumors versus other subtypes was statistically significant in both ER-positive $(p=0.001)$ and ER-negative $(p<0.001)$ tumors, demonstrating a benefit in all patients with HER2E tumors regardless of HR status. Also, a study evaluating lapatinib and letrozole in first-line HR-positive breast cancer showed that patients with HER2-negative/HER2E tumors benefited from lapatinib therapy [25]. These data suggest that there is value in intrinsic subtyping of ER-positive tumors.

Individual gene expressions from the PAM50 panel associated with pCR were $E R B B 2$ and $E S R 1$ : higher $E R B B 2$ level and lower ESRI level were associated with increasing pCR rate. This finding is consistent with RNA sequencing data from CALGB 40601 [6] and NeoALLTO [13].

In the NSABP B-41 primary analysis, dual HER2 targeting with trastuzumab plus lapatinib did not statistically significantly increase the pCR rates in the breast or breast plus nodes as compared to trastuzumab alone [5]. Results of the current analysis are consistent with this finding. While all three HER2-targeted neoadjuvant regimens produced higher pCR rates in the HER2E population as compared to other intrinsic subtypes, patients with HER2E tumors did not benefit more from dual-targeted therapy versus trastuzumab alone. As has been reported, the trastuzumab arm experienced a higher $\mathrm{pCR}$ rate than expected, which may have affected the ability of NSABP B-41 to detect a statistically significant difference between dual-targeted therapy and 
trastuzumab alone [5]. Despite the lack of lapatinib combination therapy benefit, the PAM50 analysis demonstrated an ability to identify patients more likely to benefit from both trastuzumab-based regimens evaluated.

Our study did not identify an EFS benefit among patients with HER2E tumors versus other subtypes. Interestingly, Fernandez-Martinez, et al. preliminarily reported an EFS benefit among patients with HER2E tumors who received dual-HER2-targeted trastuzumab plus lapatinb plus chemotherapy versus trastuzumab plus chemotherapy in CALGB40601, but not among patients with other subtypes. [26].

There is also evidence from other HER2 targeted therapy trials that HER2E subtype predicts pCR. Initial evidence has demonstrated a breast and pCR benefit, respectively, for dual HER 2 targeting with trastuzumab plus pertuzumab, including a differential benefit in patients with HER2E tumors [18, 27].

The 42 matched paired pre- and post-treatment samples revealed at surgery a result of HER2E subtype in $12(60.0 \%)$ and luminal A subtype in 6 (30.0\%). This pattern differed from that observed in CALGB 40601, where a majority of HERE subtype tumors demonstrated post-treatment alterations to luminal A subtype [6 of 9 (66.7\%)] after HER2targeted therapy plus chemotherapy [6]. It is possible that these differences are due to tumor heterogeneity, but posttreatment subtype alterations have important implications for treatment of residual disease.

Strengths of the current analysis include use of a large dataset with biomarkers from a randomized controlled trial and use of stringent pCR criteria (ypT0/Tis ypN0) consistent with current standards of reporting. Limitations include the limited number of patients with tumor blocks resulting in partial inclusion of study participants from the parent study and the low number of event-free and overall survival events. Meta-analyses with other similar studies will provide valuable additional information about these long-term outcomes in patients with early breast cancer.

Results of the current analysis indicate that the HER2E subtype may represent a favorable marker for predicting benefit from HER2-targeted agents, particularly trastuzumab-based therapies. Available evidence suggests a potential role for intrinsic subtype in identifying appropriate patients for neoadjuvant HER2-targeted therapy. These results also underscore the need to elucidate the role of intrinsic subtype for treatment selection in patients without HER2E tumors and identify appropriate treatment for these patients who experience low pCR rates. Prospective trials of neoadjuvant anti-HER2 therapy with randomization according to intrinsic subtype are now warranted in order to optimize treatment for patients without HER2E subtype tumors. Future directions include an ongoing meta-analysis of intrinsic subtype data to thoroughly evaluate the benefit of pCR on event-free and overall survival, and the use of proteomic analysis in combination with intrinsic subtype to further refine patient selection for specific therapies [28].

Acknowledgements The authors would like to thank the Georgetown University Core labs (Histopathology and Tissue Shared Resource [HTSR] and Genomics and Epigenomics Shared Resource [GESR]) for technical services in this project as well as the patients, investigators, and NSABP Foundation for their contributions, Phillips Gilmore Oncology Communications (Philadelphia, USA) for providing technical assistance, and T. Blaise Springfield for his assistance with figures and tables and article submission.

Funding This work is partially funded by the Aleksandr Savhcuk Foundation [S.M.S.], Breast Cancer Research Foundation [BCRF16-190; S.M.S.], and GlaxoSmithKline. This work and the HTSR and GESR are also funded in part by National Cancer Institute at the National Institutes of Health Cancer Center Support Grant to Georgetown Lombardi Comprehensive Cancer Center [P30CA051008; Site P.I. L.M. Weiner]. The authors had full access to all the data in the study and had final responsibility for the decision to submit for publication. Any opinions, findings, and conclusions expressed in this work are those of the authors and do not necessarily reflect those of the Breast Cancer Research Foundation, Aleksandr Savchuk Foundation, GlaxoSmithKline, the National Cancer Institute at the National Institutes of Health, or National Institutes of Health.

Data availability The datasets during and/or analyzed during the current study are available from the corresponding author on reasonable request.

\section{Compliance with ethical standards}

Conflict of interest S.M.S. reports remuneration from Bristol-Myers Squibb, Caris Life Sciences, Daiichi-Sankyo, Eli Lilly \& Co., Genentech/Roche, NanoString Technologies, Novartis, AstraZeneca for participation on IDMC; and support for third-party writing assistance outside the conduct of the study furnished by Daniel Clyde, $\mathrm{PhD}$ of Health Interactions; consultant/advisory role with Cardinal Health, Daiichi-Sankyo, Eli Lilly \& Co., Genentech/Roche, Genomic Health, Inivata, Peiris Pharmaceuticals, and Tocagen; and funding from the Aleksandr Savchuk Foundation, Breast Cancer Research Foundation, and Genentech. G.T. reports consultant/advisory role with Georgetown University. P.C.L. reports consultant/advisory role with Bayer/ Loxo Pharmaceuticals and stock ownership in Amgen (AMGN). A.R. reports consultant/advisory role with AstraZeneca and Apobiologix and funding from Merck. C.E.G. reports remuneration from Abbvie, Daiichi-Sankyo, Genentech/Roche and funding from GlaxoSmithKlein. P.R. reports remuneration from AstraZeneca, Genentech/Roche, and Eli Lilly \& Co. E.P.M. reports consultant/advisory role with Biotheranostics, Daiichi-Sankyo, Genentech/Roche, Genomic Health, and Merck. N.W. reports funding from the Breast Cancer Research Foundation. All other authors report no conflicts of interest.

Ethical approval All procedures performed in studies involving human participants were in accordance with the ethical standards of the institutional and/or national research committee and with the 1964 Helsinki declaration and its later amendments or comparable ethical standards. 
Informed consent Informed consent for usage of tissue samples for future research was obtained from all individual participants included in the study.

Open Access This article is distributed under the terms of the Creative Commons Attribution 4.0 International License (http://creativeco mmons.org/licenses/by/4.0/), which permits unrestricted use, distribution, and reproduction in any medium, provided you give appropriate credit to the original author(s) and the source, provide a link to the Creative Commons license, and indicate if changes were made.

\section{References}

1. Swain SM, Baselga J, Kim SB et al (2015) Pertuzumab, trastuzumab, and docetaxel in HER2-positive metastatic breast cancer. N Engl J Med 372:724-734

2. Cameron D, Piccart-Gebhart MJ, Gelber RD et al (2017) 11 years' follow-up of trastuzumab after adjuvant chemotherapy in HER2positive early breast cancer: final analysis of the HERceptin Adjuvant (HERA) trial. Lancet 389:1195-1205

3. Cortazar P, Zhang L, Untch M et al (2014) Pathological complete response and long-term clinical benefit in breast cancer: the CTNeoBC pooled analysis. Lancet 384:164-172

4. Baselga J, Bradbury I, Eidtmann $\mathrm{H}$ et al (2012) Lapatinib with trastuzumab for HER2-positive early breast cancer (NeoALTTO): a randomised, open-label, multicentre, phase 3 trial. Lancet 379:633-640

5. Robidoux A, Tang G, Rastogi P et al (2013) Lapatinib as a component of neoadjuvant therapy for HER2-positive operable breast cancer (NSABP protocol B-41): an open-label, randomised phase 3 trial. Lancet Oncol 14:1183-1192

6. Carey LA, Berry DA, Cirrincione CT et al (2016) Molecular heterogeneity and response to neoadjuvant human epidermal growth factor receptor 2 targeting in CALGB 40601, a randomized phase III trial of paclitaxel plus trastuzumab with or without lapatinib. J Clin Oncol 34:542-549

7. Hicks M, Macrae ER, Abdel-Rasoul M et al (2015) Neoadjuvant dual HER2-targeted therapy with lapatinib and trastuzumab improves pathologic complete response in patients with early stage HER2-positive breast cancer: a meta-analysis of randomized prospective clinical trials. Oncologist 20:337-343

8. Clavarezza M, Puntoni M, Gennari A et al (2016) Dual block with lapatinib and trastuzumab versus single-agent trastuzumab combined with chemotherapy as neoadjuvant treatment of HER2positive breast cancer: a meta-analysis of randomized trials. Clin Cancer Res 22:4594-4603

9. Robidoux A, Tang G, Rastogi P et al (2016) Evaluation of lapatinib as a component of neoadjuvant therapy for HER2 + operable breast cancer: five year outcomes of NSABP protocol B-41. J Clin Oncol 34:501

10. Parker JS, Mullins M, Cheang MCU et al (2009) Supervised risk predictor of breast cancer based on intrinsic subtypes. J Clin Oncol 27:1160-1167

11. Dieci MV, Prat A, Tagliafico E et al (2016) Integrated evaluation of PAM50 subtypes and immune modulation of pCR in HER2-positive breast cancer patients treated with chemotherapy and HER2-targeted agents in the CherLOB trial. Ann Oncol 27:1867-1873

12. Prat A, Bianchini G, Thomas M et al (2014) Research-based PAM50 subtype predictor identifies higher responses and improved survival outcomes in HER2-positive breast cancer in the NOAH study. Clin Cancer Res 20:511-521

13. Fumagalli D, Venet D, Ignatiadis $M$ et al (2017) RNA sequencing to predict response to neoadjuvant anti-HER2 therapy: a secondary analysis of the NeoALTTO randomized clinical trial. JAMA Oncol 3:227-234

14. McShane LM, Altman DG, Sauerbrei W et al (2005) Reporting recommendations for tumor marker prognostic studies (REMARK). J Natl Cancer Inst 97:1180-1184

15. Jørgensen CL, Nielsen TO, Bjerre KD et al (2014) PAM50 breast cancer intrinsic subtypes and effect of gemcitabine in advanced breast cancer patients. Acta Oncol 53:776-787

16. Benjamini Y, Hochberg Y (1995) Controlling the false discovery rate: a practical and powerful approach to multiple testing. J R Stat Soc Series B Stat Methodol 57:289-300

17. Hommel G (1988) A stagewise rejective multiple test procedure based on a modified Bonferroni test. Biometrika 75:383-386

18. Akaike H (1974) A new look at the statistical model identification. IEEE Trans Automat Contr 19:716-723

19. Gianni L, Pienkowski T, Im Y-H et al (2012) Efficacy and safety of neoadjuvant pertuzumab and trastuzumab in women with locally advanced, inflammatory, or early HER2-positive breast cancer (NeoSphere): a randomised multicentre, open-label, phase 2 trial. Lancet Oncol 13:25-32

20. Cejalvo JM, Pascuala T, Fernández-Martíneze A et al (2018) Clinical implications of the non-luminal intrinsic subtypes in hormone receptor-positive breast cancer. Cancer Treat Rev 67:63-70

21. Llombart-Cussac A, Cortés J, Paré L et al (2017) HER2-enriched subtype as a predictor of pathological complete response following trastuzumab and lapatinib without chemotherapy in early-stage HER2-positive breast cancer (PAMELA): an open-label, singlegroup, multicentre, phase 2 trial. Lancet Oncol 18:545-554

22. Buzdar AU, Ibrahim NK, Francis D et al (2005) Buzdar significantly higher pathologic complete remission rate after neoadjuvant therapy with trastuzumab, paclitaxel, and epirubicin chemotherapy: results of a randomized trial in human epidermal growth factor receptor 2-positive operable breast cancer. J Clin Oncol 23:3676-3685

23. Prat A, Pascual T, De Angelis C et al (2019) HER2-enriched subtype and ERBB2 expression in HER2-positive breast cancer treated with dual HER2 blockade. J Natl Cancer Inst. https://doi. org/10.1093/jnci/djz042

24. Guarneri V, Frassoldati A, Bottini A et al (2012) Preoperative chemotherapy plus trastuzumab, lapatinib, or both in human epidermal growth factor receptor 2-positive operable breast cancer: results of the randomized phase II CHER-LOB study. J Clin Oncol 30:1989-1995

25. Prat A, Cheang MCU, Galvan P et al (2016) Prognostic value of intrinsic subtypes in hormone receptor- positive metastatic breast cancer treated with letrozole with or without lapatinib. JAMA Oncol 2:1287-1294

26. Fernandez-Martinez A, Tanioka M, Fan C et al (2019) Genomicbased predictive biomarkers to anti-HER2 therapies: a combined analysis of CALGB 40601 (Alliance) and PAMELA clinical trials. J Clin Oncol 37:571

27. Swain SM, Ewer MS, Viale G et al (2018) Pertuzumab, trastuzumab, and standard anthracycline- and taxane-based chemotherapy for the neoadjuvant treatment of patients with HER2-positive localized breast cancer (BERENICE): a phase II, open-label, multicentre, multinational cardiac safety study. Ann Oncol 29:646-653

28. Johansson HJ, Socciarelli F, Vacanti NM et al (2019) Breast cancer quantitative proteome and proteogenomic landscape. Nat Commun 10:1600. https://doi.org/10.1038/s41467-019-09018-y

Publisher's Note Springer Nature remains neutral with regard to jurisdictional claims in published maps and institutional affiliations. 


\section{Affiliations}

Sandra M. Swain ${ }^{1,2} \odot$ - Gong Tang ${ }^{1,3} \cdot$ Peter C. Lucas $^{1,3} \cdot$ André Robidoux $^{1,4} \cdot$ David Goerlitz $^{2} \cdot$ Brent T. Harris $^{2}$. Hanna Bandos ${ }^{1,3}$. Charles E. Geyer Jr. ${ }^{1,5}$. Priya Rastogi ${ }^{1,3,6}$. Eleftherios P. Mamounas ${ }^{1,7}$. Norman Wolmark ${ }^{1,3}$

1 National Surgical Adjuvant Breast and Bowel Project (NSABP), Nova Tower 2, 100 Allegheny Square, Pittsburgh, PA 15212, USA

2 Georgetown Lombardi Comprehensive Cancer Center, Georgetown University Medical Center, 4000 Reservoir Road NW, 120 Building D, Washington, DC 20057, USA

3 University of Pittsburgh, 4200 Fifth Ave, Pittsburgh, PA 15260, USA

4 Centre Hospitalier de L'Université de Montréal, 3840 St Urbain St, Montreal, QC H2W 1T8, Canada
5 Massey Cancer Center, Virginia Commonwealth University, 401 College Street, Box 980037, Richmond, VA 23298, USA

6 Magee-Womens Hospital, 300 Halket Street, Pittsburgh, PA 15213, USA

7 Orlando Health UF Health Cancer Center, 1400 S Orange Avenue, Orlando, FL 32876, USA 